\title{
Skin Bends: a Cutaneous Manifestation of Decompression Sickness
}

\author{
Ronald S. Strauss, M.D.
}

Internal Medicine, Private Medical, San Francisco, CA, USA

$\mathrm{J}$ Gen Intern Med 34(10):2290

DOI: $10.1007 /$ s11606-019-05208-y

(C) Society of General Internal Medicine 2019

\begin{abstract}
A
62-year-old man reported the rapid onset of acute ataxia and a pruritic, tender, mottled rash on his torso. The patient had been on two SCUBA dives a few hours earlier-as deep as $20 \mathrm{~m}$, with adherence to standard ascent protocols. He denied vision changes, weakness, and shortness of breath. He texted photos of his skin findings to his doctor (Figures 1 and 2). A clinical diagnosis of decompression sickness (DCS) was made. Medics administered high flow oxygen and intravenous fluids. Hyperbaric treatment was not available. In the emergency department, his symptoms resolved within several hours.

Cutaneous abnormalities, commonly known as "skin bends," are among the most common findings in DCS. ${ }^{1}$
\end{abstract}

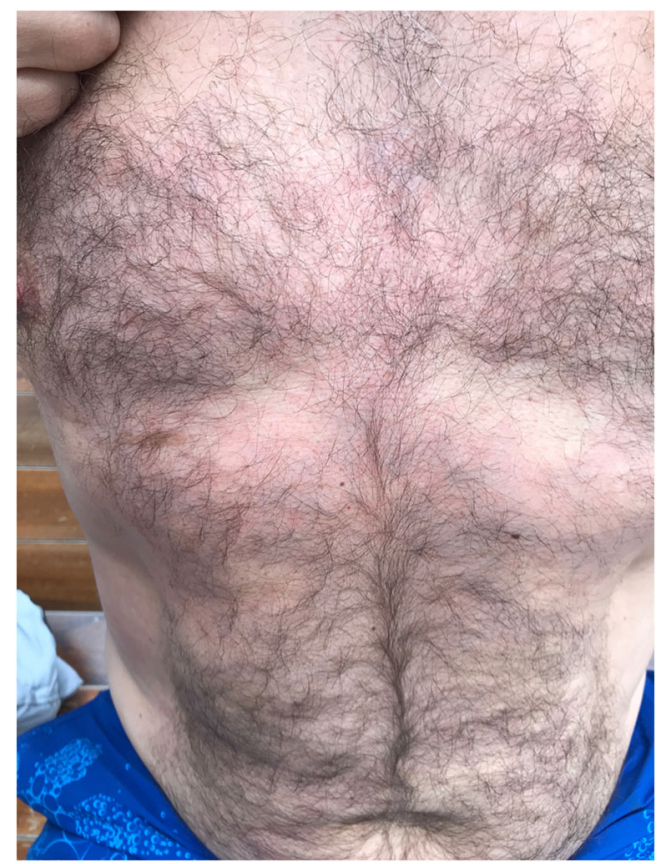

Figure 1 Photograph of mottled, tender, pruritic rash on patient's torso

Received March 4, 2019

Revised April 24, 2019

Accepted July 9, 2019

Published online July 25, 2019

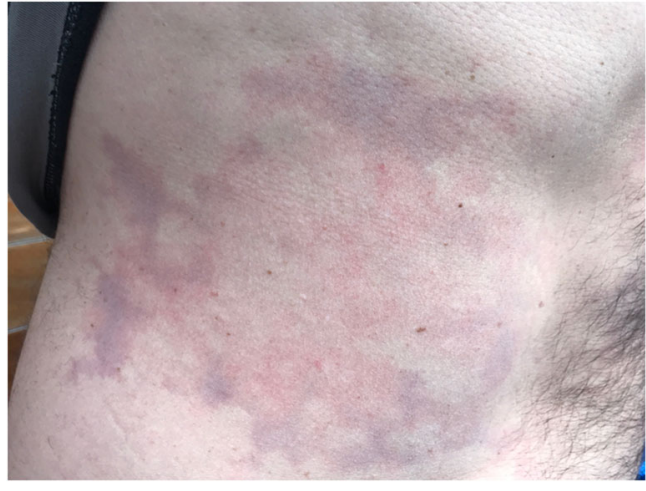

Figure 2 Another photograph of the rash on patient's torso, closer view

Cutis marmorata due to decompression sickness classically presents as an erythematous, pruritic rash that spreads irregularly and deepens in color to become mottled, cyanotic patches. ${ }^{2}$ The cause is believed to be nitrogen bubbles within tissues and vessels. DCS may result in severe complications-including spinal cord injury, pulmonary venous gas embolism, acute circulatory collapse, and stroke from paradoxical arterial embolism. The skin findings typically resolve without sequelae, but are an important, early sign of DCS and indicate the need for emergent evaluation.

Corresponding Author: Ronald S. Strauss, M.D.; Internal Medicine Private Medical, 3580 California Street, San Francisco, CA, USA (e-mail: drstrauss@privatemedical.org).

\section{Compliance with Ethical Standards:}

Conflict of Interest: The author declares that he has no conflict of interest.

\section{REFERENCES}

1. Xu W, Liu W, Huang G, Zou Z, Cai Z, Xu W. Decompression illness: clinical aspects of 5278 consecutive cases treated in a single hyperbaric unit. PLoS One. 2012;7(11):e50079.

2. Vasileios K. Cutis Marmorata in Decompression Sickness. N Engl J Med. 2010;362:23.

Publisher's Note Springer Nature remains neutral with regard to jurisdictional claims in published maps and institutional affiliations. 